\title{
Calculation of Positron Observables Using a Finite-Element-Based Approach
}

\author{
P.A. Sterne \\ J.E. Pask \\ B.M. Klein
}

This paper was prepared for submittal to the

\author{
8th International Workshop on Slow Positron Beam Techniques \\ Cape Town, South Africa \\ September 6-12, 1998
}

November 4, 1998

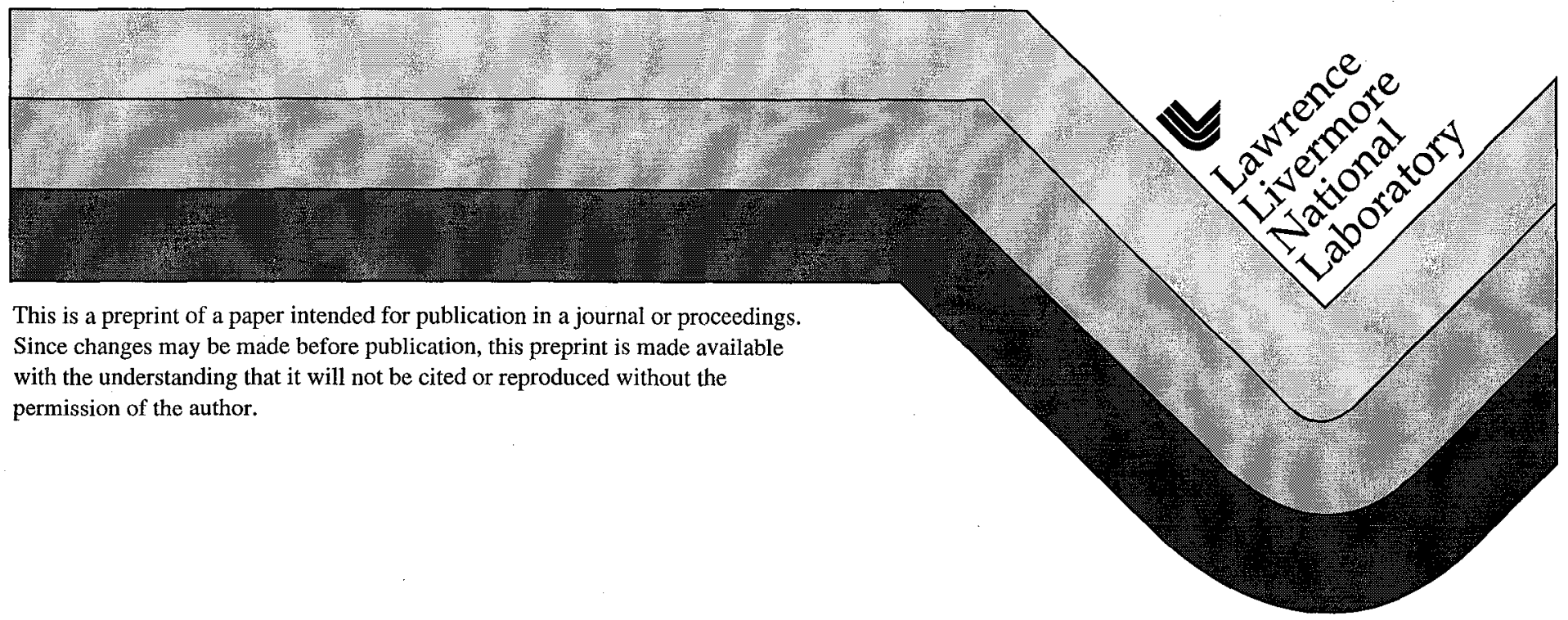




\section{DISCLAIMER}

This document was prepared as an account of work sponsored by an agency of the United States Govemment. Neither the United States Govemment nor the University of California nor any of their employees. makes any warranty, express or implied. or assumes any legal liability or responsibility for the accuracy. completeness, or usefulness of any information. apparatus, product, or process disclosed. or represents that its use would not infringe privately owned rights. Reference herein to any specific commercial product, process, or service by trade name, trademark, manufacturer, or otherwise. does not necessarily constitute or imply its endorsement, recommendation, or favoring by the United States Government or the University of California. The views and opinions of authors expressed herein do not necessarily state or reflect those of the United States Government or the University of California, and shall not be used for advertising or product endorsement purposes. 


\title{
Calculation of Positron Observables Using a Finite-Element-Based Approach
}

\author{
P.A. Sterne1,2, J.E. Pask², and B.M. Klein ${ }^{2}$ \\ 1 Lawrence Livermore National Laboratory, Livermore, CA 94550, USA \\ ${ }^{2}$ Department of Physics, Universily of California, Davis, CA 95616, USA
}

\begin{abstract}
We report the development of a new method for calculating positron observables using a finite-element approach for the solution of the Schrodinger equation. This method combines the advantages of both basis-set and real-space-grid approaches. The strict locality in real space of the finite element basis functions results in a method that is well suited for calculating large systems of a thousand or more atoms, as required for calculations of extended defects such as dislocations. In addition, the method is variational in nature and its convergence can be controlled systematically. The calculation of positron observables is straightforward due to the real-space nature of this method. We illustrate the power of this method with positron lifetime calculations on defects and defect-free materials, using overlapping atomic charge densities.

PACS: $\quad$ 78.70.B, 71.60, 02.70.D
\end{abstract}

Keywords: Finite Element Method, Positron Lifetimes, Local Density Approximation, Monovacancies, $\mathrm{C}_{60}$, Fullerenes

Corresponding Author:
P.A. Sterne

Lawrence Livermore National Laboratory

P.O. Box 808, L-415

Livermore, CA 94550

USA
Phone: +1 $925422-2510$

Fax: $\quad+1925422-6594$

Email: sterne1@1ln1.gov 


\section{Introduction}

Quantum mechanical calculations of positron states in materials provide the basis for the interpretation of positron annihilation experiments such as positron lifetimes, core-electron momentum measurements, and 2D-ACAR spectra [1]. Several methods that were originally developed for electronic structure calculations have been adapted to calculate the positron distributions and related observables in periodic systems. This has been very successful for simple metallic systems, where quantitative lifetime calculations yield results that agree with experiment to within a few picoseconds [2-4]. Nevertheless, there are significant limitations in many of these approaches. Calculations based on the linear-muffin-tin orbital (LMTO) method $[5,6]$ are well suited for electrons, but are less suitable for describing positron distributions in the interstitial region, especially for open structures that are difficult to treat in the atomic sphere approximation (ASA). In addition, traditional approaches for the solution of the Schrodinger equation result in computation times that grow with the third power of the system size, and at least quadratically in memory rcquirements. With currently available computers, practical calculations are limited to systems on the order of 100-200 atoms, which is often too small to model grain boundaries, dislocations, defects on surfaces, and other systems of current interest to the positron annihilation community. Although methods with better scaling have been developed [7], there is still a clear need for a new approach that is suitable for very large systems and open structures with arbitrary geometry.

This need has prompted us to develop a new method for calculating positron observables based on a finite-element (FE) approach which combines the favorable properties of both basis-set and real-space-grid approaches. This method is capable of treating very large systems of thousands of atoms with arbitrary accuracy. The method is a full-potential approach that makes no approximations to the shape of the positron potential, in contrast to ASA-based approaches like the LMTO. In this paper, we present a brief description of this method together with results of calculations on positron distributions and lifetimes in elemental metals, vacancies, and ordered pure and potassium-doped fullerenes. Our results demonstrate that this new method is very well suited for positron calculations and already provides reliable results for very large systems.

\section{Method}

The finite element method has a long history of success in diverse applications ranging from civil engineering to quantum mechanics [8,9]. 'There have been a number of applications to the electronic structure of atoms and molecules [10,11], but only a few to periodic solids [12-14]. We have developed a full 3-D finite-element-based approach to the solution of the Schrodinger equation with boundary conditions appropriate to a periodic solid. This approach 
is developed in detail in reference [14]. Here, we summarize the approach and discuss its adaptation to positron calculations.

In the FE method, solutions to differential equations (e.g. electron or positron wavefunctions) are described in terms of strictly local, piecewise polynomial basis functions. The unit cell is partitioned into subdomains called elements. Local polynomials are defined in each element and these polynomials are linked continuously to neighboring elements and across the domain boundaries to create piecewise continuous functions. These piecewise continuous polynomials form the basis functions of our approach. Expansions in terms of these basis functions can be used to describe an arbitrary function throughout the unit cell, and the accuracy of this representation can be improved systematically by increasing the order of the polynomials or the number of elements. The method therefore has all the advantages and flexibility of a variational basis-set method. In addition, the piecewise continuous basis functions are strictly local in real-space, which results in sparse Hamiltonian and overlap matrices and enables the efficient use of iterative diagonalization techniques and parallel computer architectures. The method therefore combines both the computational advantages of real-space-grid methods and the variational nature and convenient expansion properties of basis-set approaches.

The number of elements required for an accurate calculation depends on the rapidity of wavefunction variation across the unit cell. Positrons typically occupy the interstitial region of the solid, with relatively modest variations in wavefunction across the unit cell, so we might expect that positron calculations can be performed fairly accurately with a small number of elements. Electron calculations with an empirical Si pseudopotential yield a band structure in excellent agreement with highly-converged plane-wave calculations with only $6 \times 6 \times 6$ elements in the unit cell [14]. It is reasonable to expect similar, or even slightly better convergence for the positron calculations, and this is confirmed by our results reported below.

The FE method needs a positron potential in order to calculate the positron wavefunction. This potential is currently obtained in our approach from overlapping atomic calculations. The electrostatic interactions with the electrons and the nuclei provide the dominant part of the potential, and this is obtained by overlapping atomic electrostatic potentials. The remaining electron-positron correlation potential is taken from a local-densityapproximation (LDA) form [2] where the potential depends only on the electron charge density, which is also obtained by overlapping atomic charge densities. We have used both an LDA form [2] based on free-electron calculations by Arponen and Pajanne [16] and a more recent modification using a Generalized Gradient Approximation (GGA) [4]. The overlapping atomic charge density approach [17] has been used successfully to treat fairly large systems (of order 256 atoms) in finite-difference calculations of positrons in solids [18]. At present, our positron 
calculations do not allow for electron or positron charge self-consistency, although this is planned for the future. This lack of self-consistency and uncertainties in the electron-positron interactions are the most significant limitations of the current implementation.

Given a positron potential, the FE method can solve the Schrodinger equation for the lowest-energy positron state, and the positron charge density is obtained directly in real-space from the wavefunction expansion. The positron lifetime depends on the product of the electron and positron charge densities integrated over the unit cell together with an enhancement function that depends on the total electron density [1-4]. The enhancement function is again obtained from an LDA [1-3] or GGA [4] form.

Matrix elements and other integrals over the unit cell such as positron lifetimes are easy to calculate in the FE approach, since the basis functions have a simple polynomial form, and resulting wavefunctions and related quantities are uniquely defined at all points in the unit cell in terms of the polynomial coefficients in the wavefunction expansion. This simple expansion property and the direct real-space nature of the method makes the calculation of positron observables straightforward. The current implementation provides positron distributions and lifetimes, and we plan to add calculation of electron-positron momentum densities in the near future.

\section{Positron lifetimes in metals and monovacancies}

Calculations of positron lifetimes in elemental metals provide a useful test of the reliability of any new method for positron calculations. Figure 1 shows a comparison between positron lifetimes calculated in the FE-LDA approach and those obtained using a traditional LMTO-ASA approach, both with the same local density form for the potential [2]. The corresponding FE, LMTO, and experimental lifetimes are given in Table 1, together with FE results using the GGA potential. The FE-LDA approach yields lifetimes that are in very good overall agreement with the LMTO method, although they tend to be slightly longer by about 15 ps. This may be due to differences in the calculational details: the FE calculations are nonself-consistent while the LMTO calculations are based on self-consistent charge densities, and the FE calculations make no approximations to the shape of the potential while the LMTO calculations use the ASA. The calculations were performed for $4 \times 4 \times 4-, 6 \times 6 \times 6-$, and $8 \times 8 \times 8-$ element meshes. In all cases the calculated lifetimes were essentially converged for the $4 \times 4 \times 4$ calculation, with changes in lifetimes of less than $0.5 \%$ and typically on the order of $0.1 \%$ with increasing numbers of elements. The most highly converged calculations $(8 \times 8 \times 8)$ are reported in Table 1.

The GGA results in Table 1 are in reasonable agreement with the calculations of Ref. [4] which used the same GGA functional within a finite difference method based on atomic 
superposition. There is excellent agreement for many of the elements, but there are significant differences for some transition metals which may be due to the choice of electronic configuration in the atomic calculations. Ref. [4] used atomic ground state configurations while the present calculation used configurations that more closely reflect the electronic structure of the solid. The overall agreement is still very good and confirms the accuracy the method for positron lifetime calculations.

Table 1 also lists results for monovacancy lifetimes in $\mathrm{Al}, \mathrm{Cu}$, and $\mathrm{Ag}$ based on 31-atom supercells. The agreement with experiment is very good, although the FE method slightly underestimates the experimental lifelime values. The calculated values increase slightly to give better agreement with experiment when larger supercell sizes are used for the monovacancy calculations.

It is known that calculated monovacancy lifetimes are sensitive to the supercell size [18], and the 31-atom calculations listed in table 2 are not quite converged with respect to supercell size. We have examined the convergence of the Al monovacancy with respect to both supercell size and the number of elements per unit cell. The results are shown in table 2. Note that the system is well converged to a value of $243 \mathrm{ps,} \mathrm{very} \mathrm{close} \mathrm{to} \mathrm{the} \mathrm{experimental} \mathrm{value} \mathrm{of}$ 244 ps, with 16 elements per primitive unit cell and a 255 atom supercell. This convergence is confirmed by the absence of any further change in the lifetime upon increasing either the number of elements or the supercell size, or both. The largest supercell calculations for an 863atom unit cell were well within the range of out FE implementation, indicating that the method is well suited for problems that require large system sizes, such as dislocations and other extended defects.

\section{Positrons in potassium-doped fullerenes}

The FE method has three significant advantages over our previous LMTO-ASA method. First, it is capable of treating very large systems, as illustrated by the 863-atom unit cell calculations for an $\mathrm{Al}$ monovacancy. Second, it is well suited for treating open structures where the atomic sphere approximation may give a poor description of the electron and positron charge distributions. Third, it provides a full three-dimensional representation of the positron distribution in real space, in contrast the the LMTO which distorts the representation of space through the atomic sphere approximation. All three of these advantages are illustrated here by a set of positron calculations on the low temperature structure of solid $\mathrm{C}_{60}$ with and without potassium doping.

Solid $\mathrm{C}_{60}$ fullerene forms a face-centered cubic structure at higher temperatures with the carbon fullerene molecules randomly oriented with respect to each other. At lower temperatures, the molecules orient to form a simple cubic structurc consisting of four $C_{60}$ 
molecules to give a 240 atom unit cell. Potassium doping first fills the octahedral sites between the fullerene molecules $\left(\mathrm{KC}_{60}\right)$ and with increasing doping, the tetrahedral sites are also occupied by potassium atoms $\left(\mathrm{K}_{3} \mathrm{C}_{60}\right)$, resulting in a compound that exhibits high temperature superconductivity[19]. Puska and Nieminen [20] have performed positron calculations on this system using a finite difference method [17]. They used a simplified structure for the $\mathrm{C}_{60}$ orientation so that they could treat the system as face-centered cubic with a single fullerene molecule. We compare their results with our calculations based on the larger 240-atom simple cubic structure.

Puska and Nieminen obtained lifetime values of 327, 276, and 261 picoseconds for $\mathrm{C}_{60}$, $\mathrm{KC}_{60}$, and $\mathrm{K}_{3} \mathrm{C}_{60}$, respectively. Using a similar LDA enhancement and correlation function, we calculate corresponding lifetime values of 330, 273, and 261 picoseconds, in excellent agreement with the earlier results.

Calculations of the positron distribution indicate that the positron does not enter the fullerene molecule, but prefers to sit mostly in the octahedral site and partly in the tetrahedral site between molecules [20]. Potassium also preferentially occupies these octahedral sites, so the repulsion between the potassium nucleus and the positron will force a redistribution of the positron charge density in the material with doping. This is illustrated in Figure 2. With no potassium, the positron charge is localized in the octahedral site, and avoids the open space at the center of the fullerene molecule. Once the octahedral site is occupied by a potassium atom $\left(\mathrm{KC}_{60}\right)$, the positron concentrates in the tetrahedral sites, still avoiding the centers of the fullerene molecules. When potassium atoms occupy the tetrahedral sites as well, the positron squeezes between the tetrahedral and octahedral site, and also shows a small buildup inside the fullerene molecule. The calculated positron lifetime changes with the potassium doping due to the redistribution of the positron charge density and the resulting change in the electronpositron charge density overlap.

\section{Conclusions}

We have successfully applied a new finite element electronic structure technique to calculate positron distributions and lifetimes in a variety of systems including elemental metals, vacancies in metals, and fullerene structures. Our FE approach has demonstrated its efficiency and applicability to very large systems by calculating the positron lifetime in an 863-atom monovacancy structure, and larger calculations are feasible. The method is also well suited to calculations on open structures, as demonstrated by the results on potassium-doped fullerenes. The method readily provides positron distributions in real space that facilitate an understanding of the positron sensitivity to specific parts of the unit cell, and the polynomial basis functions make it casy to calculate positron observables such as lifetimes and momentum 
densities in real space. This approach is already a valuable method for performing positron calculations on very large systems, and promises to become even more powerful once charge selfconsistency is implemented.

\section{Acknowledgments}

This work was performed under the auspices of the U.S. Department of Energy by Lawrence Livermore National Laboratory under contract number W-7405-ENG-48. 


\section{References}

[1] M. Puska and R.M. Nieminen, Rev. Mod. Phys. 66, 841 (1994)

[2] P.A. Sterne and J.H. Kaiser, Phys. Rev. B 43, 13892 (1991)

[3] M. Puska, J. Phys. Condens. Matter, 3, 3455 (1991)

[4] B. Barbiellini, M. Puska, T. Korhonen, A. Harju, T. Torsti and R.M. Nieminen, Phys. Rev. B 53, 16201 (1996)

[5] O.K. Andersen, Phys. Rev. B 12, 3060 (1975)

[6] H.L. Skriver, The LMTO Method, Springer Verlag, New York, 1984

[7] A.P. Seitsonen, M.J. Puska and R.M. Nieminen, Phys. Rev. B 51, 14057 (1995)

[8] D.S. Burnett, Finite Element Analysis, Addison-Wesley, Reading, 1987

[9] G. Strang and G.J. Fix, An Analysis of the Finite Element Method, Prentice-Hall, Englewood Cliffs, 1973

[10] J. Linderberg, Comp. Phys. Rep. 6, 209 (1987)

[11] S.R. White, J.W. Wilkins, and M.P Teter, Phys. Rev. B 39, 5819 (1989)

[12] E. Tsuchida and M. Tsukada, Phys. Rev B 52, 5573 (1995); Phys. Rev. B 54, 7602 (1996)

[13] B. Hermansson and D. Yevick, Phys. Rev. B 33, 7241 (1986)

[14] J.E. Pask, B.M. Klein, C.Y. Fong and P.A. Sterne, submitted to Physical Review B

[15] Finite Element Handbook, Eds. H. Kardestuncer, D.H. Norrie, et al., McGraw-Hill, New York, 1987, p. 2.126

[16] J. Arponen and E. Pajannc, Ann. Phys. (N.Y.) 121, 343 (1979)

[17] M.J. Puska and R.M. Nieminen, J. Phys. F: Metal Phys. 13, 333 (1983)

[18] T. Korhonen, M.J. Puska and R.M. Nieminen, Phys. Rev. B 54, 15016 (1996)

[19] A.F. Hebard, M.J. Rosseinsky, R.C. Haddon, D.W. Murphy, S.H. Glarum, T.T.M. Palstra, A.P. Ramirez, and A.R. Kortan, Nature, 350, 600 (1991)

[20] M.J. Puska and R.M. Nieminen, J. Phys. Condensed Matt. 4, L149 (1992) 


\section{Figure Captions}

1. Comparison of positron lifetimes (in picoseconds) calculated in the LMTO-ASA and FE methods, using the same LDA form for the electron-postron correlation and enhancement. See Table 1 for more details.

2. Positron charge distributions along the simple cubic (111) direction for potassium doped fullerenes: (a) $\mathrm{C}_{60}$, (b) $\mathrm{KC}_{60}$, (c) $\mathrm{K}_{3} \mathrm{C}_{60}$. Octahedral and tetrahedral sites are indicated and the end points are located at the centers of fullerene molecules. 


\section{Tables}

Table 1.

Positron lifetimes in picoseconds for selected elemental metals and monovacancies. LMTO calculations are taken from reference [2] using the same LDA electron-positron correlation potential and enhancement function as the present FE-LDA calculations. The GGA calculations use the functional form described in Ref [4] with alpha $=0.22$. Monovacancy calculations were performed with unrelaxed atomic positions.

$\begin{array}{lrrrr}\text { Element } & \text { LMTO } & \text { FE-LDA } & \text { FE-GGA } & \text { Expt. } \\ \mathrm{Li} & 295 & 296 & 283 & 291 \\ \mathrm{Na} & 319 & 321 & 341 & 338 \\ \mathrm{~K} & 363 & 365 & 400 & 397 \\ \mathrm{Rb} & 372 & 374 & 418 & 406 \\ \mathrm{Cs} & 385 & 385 & 434 & 418 \\ \mathrm{Al} & 163 & 168 & 159 & 163 \\ \mathrm{~Pb} & 187 & 191 & 213 & 194 \\ \mathrm{Ti} & 143 & 152 & 164 & 147 \\ \mathrm{~V} & 115 & 121 & 132 & 130 \\ \mathrm{Cr} & 101 & 103 & 110 & 120 \\ \mathrm{Fe} & 101 & 107 & 120 & 106 \\ \mathrm{Ni} & 97 & 98 & 108 & 110 \\ \mathrm{Cu} & 103 & 107 & 119 & 110 \\ \mathrm{Zr} & 156 & 161 & 162 & 165 \\ \mathrm{Nb} & 121 & 125 & 128 & 119 \\ \mathrm{Mo} & 104 & 109 & 112 & 103 \\ \mathrm{Pd} & 103 & 108 & 122 & 96 \\ \mathrm{Ag} & 120 & 127 & 149 & 131 \\ \mathrm{Ta} & 116 & 123 & 130 & 116 \\ \mathrm{~W} & 100 & 104 & 108 & 105 \\ \mathrm{Pt} & 96 & 98 & 106 & 99 \\ \mathrm{Au} & 108 & 114 & 131 & 117 \\ \mathrm{Gd} & 199 & 204 & 213 & 230 \\ \mathrm{Vacancies} & & & & \\ \mathrm{Cu} & 164 & 169 & --- & 173 \\ \mathrm{Al} & 234 & 239 & --- & 244 \\ \mathrm{Ag} & 194 & 199 & -- & 198\end{array}$


Table 2.

Convergence results for a monovacancy in Al. The calculated lifetimes are in picoseconds. The horizontal axis gives the number of elements per primitive unit cell. For the 255 atom case, 16 elements/unit cell corresponds to a $16 \times 16 \times 16$ calculation in the supercell.

number of atoms

31

255

863
Elements/unit cell

16

54

$242 \quad 240$

$245 \quad 243$

$245 \quad 243$
239

243

243 


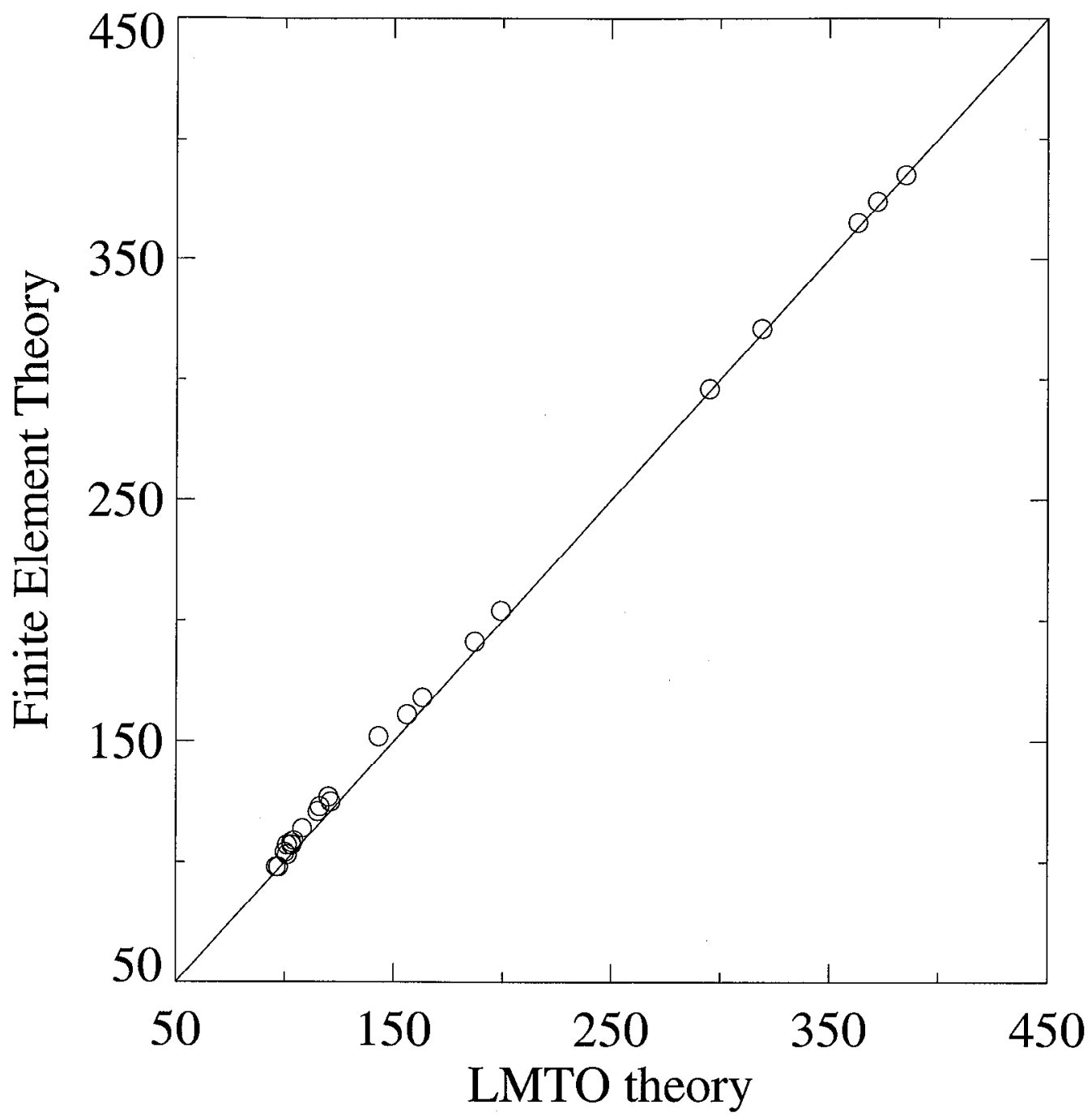

Fig 1

sterne 


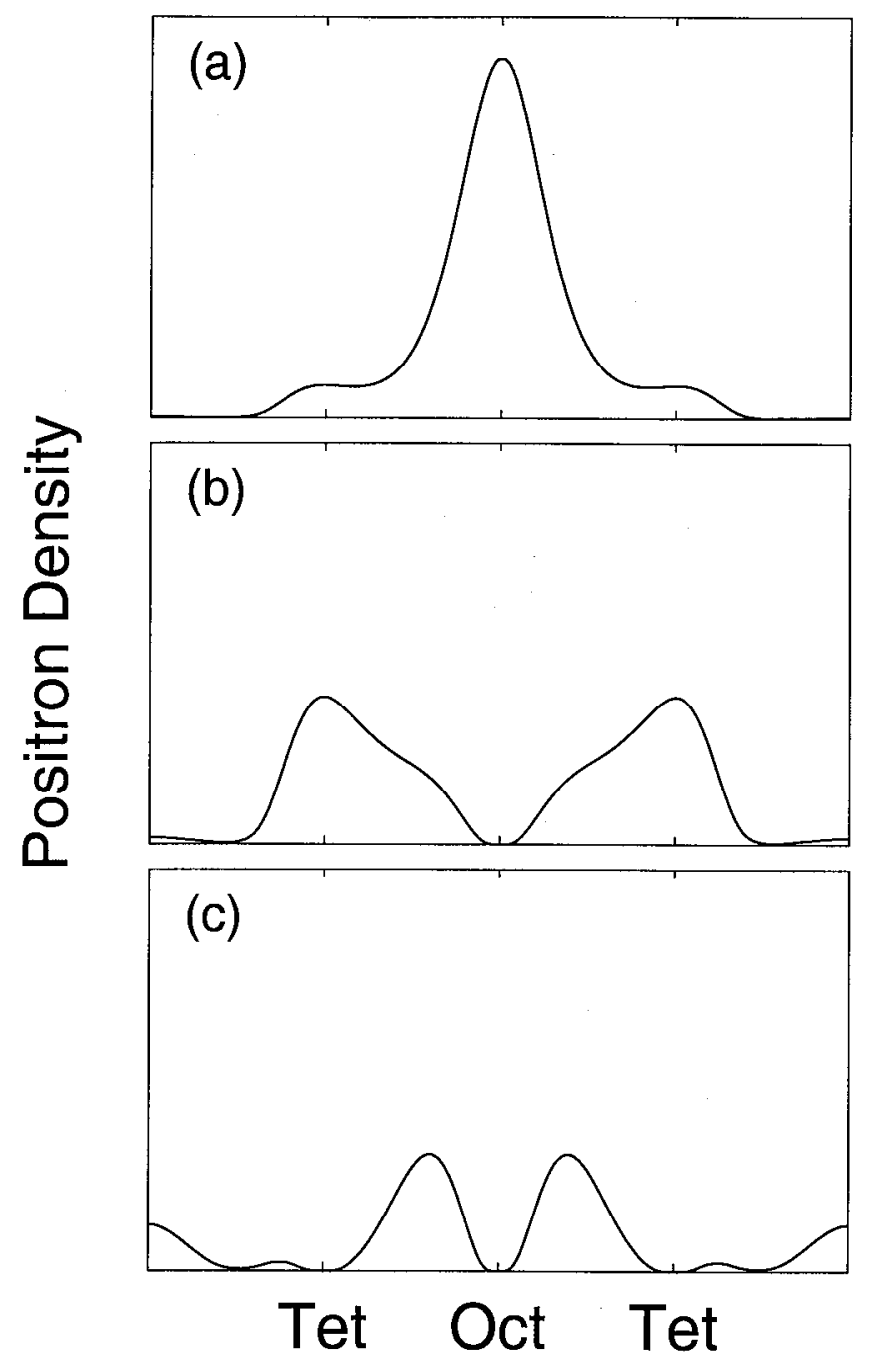

Fig 2

Sterne 